\title{
Agent Administration
}

National Cancer Institute

\section{Source}

National Cancer Institute. Agent Administration. NCI Thesaurus. Code C70962.

The act of the dispensing, applying, or tendering a medical product or other agent. 